\title{
Simulating complex networks in phase space: Gaussian boson sampling
}

\author{
Peter D. Drummond, Bogdan Opanchuk, A. Dellios and M. D. Reid \\ Centre for Quantum Science and Technology Theory, \\ Swinburne University of Technology, Melbourne 3122, Australia
}

\begin{abstract}
We show how phase-space simulations of quantum states in a linear photonic network permit the verification of measurable probabilities and entanglement. We compare our predictions with recent Gaussian boson sampling experiments of Zhong et al. These use squeezed inputs and efficient "on-off" detectors, with up to 76-th order measured coincidence counts in the data. We introduce a general definition of grouped "on-off" detection probabilities for this purpose. The positive- $P$ phase-space method is used to compute any grouped or marginal click probabilities. Additional decoherence is included to obtain agreement between theory and experiment. The only limitation in estimating grouped probabilities is the computational sampling error, which is similar in magnitude to the experimental sampling error. The results obtained and graphed here are from first-order up to 16 000-th order grouped count probabilities. However, any order between these is also computable. We extend these results to include grouped probabilities with multidimensional outcomes that have a polynomial number of points. We also analyze quadrature detection experiments, and show how to simulate genuine multipartite entanglement using Wigner phase-space methods.
\end{abstract}

\section{INTRODUCTION}

Bosonic quantum networks are increasingly useful in quantum technology and quantum computing applications [1. Linear networks driven either by nonclassical number state [2 8] or Gaussian inputs [9 12] for boson sampling are becoming widely available. The squeezedstate interferometer, which is a two-mode linear network, is being employed to enhance gravitational-wave detection sensitivity [13, 14]. More complex photonic networks are under development, both as novel interferometers [15, 16] and as test-beds for multipartite entanglement [17-19]. Other examples include the Ising machine, used to solve large $N P$-hard optimization problems $[20-22]$.

A dramatic increase in scale of a boson sampling quantum network has recently been achieved. Zhong et al. 23] implemented a 100-mode Gaussian boson sampler (GBS) with squeezed inputs, and detected the output photon counts, whose distribution is called the 'Torontonian'. They measured up to 76-th order coincidence counts in the outputs, which they estimated to take 0.6 billion years to simulate conventionally on the world's fastest current supercomputer. This has led to reports of quantum supremacy by Zhong et al.: that is, a quantum device implementing a computational task that is not classically feasible 24, 25. Similar reports have been made using quantum logic gates [26.

There is an ongoing debate on how to rigorously validate such technology [27, 28]. Validation based on loworder correlations [29] or direct classical simulation may be susceptible to mock-ups. However, Gaussian inputs to linear networks [9, 30, have a non-computable discrete count probability for large mode number. The output distribution is a Hafnian [10] for photon-numberresolving detectors. In the "on-off" or saturable detector case, it is the Torontonian [11]. Quesada et al. [11, 31] explained that classical evaluation of the ideal "Torontonian" distribution is exponentially complex, making it nearly impossible for more than 50 modes [32].
Since a direct simulation of the output correlations at large $M$ cannot be achieved in less than exponential time, another approach is needed to compare theory with experiment in a reasonable timeframe. Theoretical benchmarks are essential, both to know what output is expected, and to understand any other physics.

The objective of this paper is to do simply this: to investigate whether the theory agrees with experiment. We show how one may in part solve this problem, by simulating Gaussian boson linear networks in quantum phase space. This provides a way to verify the output quantum correlations and marginal probabilities, both in the idealized case and with other known physics included. It is important to note that we do not simulate the experiment directly using discrete photon counts [11, 31]. Rather, we verify observable, grouped probabilities by averaging over many random trajectories in phase space [33, which have the same correlations and marginal probabilities.

By contrast, an explicit simulation with discrete counts is feasible, but is currently limited to either small networks or large decoherence [34. Our methods provide a way to certify the measurable probabilities of experimental outputs, even for systems much larger than the current experimental ones. The phase space approach uses the correctness of quantum mechanics under a change of basis state to develop an alternative, powerful algorithm for efficient verification of any grouped count distribution. We have computed all of the grouped probabilities measured by Zhong et al. [23, including 76-th order coincidence counts, as well as other marginal probabilities in their data. We calculate these from the model of the apparatus and experimental parameters measured by the experimentalists.

Differences between theory and experiment appear to be caused by input pulse decoherence effects. Our phasespace simulations agree much better with experimental count distributions [23] after including these decoherence effects, and are scalable. Chi-squared tests were carried out [35, 36], which show an improvement of three orders 
of magnitude for theoretical agreement with experiment.

Results are also obtained for up to 16000 mode devices. Computational times and sampling errors are comparable to those in experiments for the same number of samples, making them useful for validation of GBS experiments. We computed marginal, low-order probabilities, as well as higher order, measurable grouped probabilities. Our methods can also be extended if necessary to include other known physics, including multiple frequency modes, dispersion, nonlinearity, decoherence and Raman/Brillouin scattering [37, allowing better understanding of these experiments.

Boson sampling outputs are exponentially hard to generate numerically. Here, we demonstrate how measurable grouped probabilities can be verified. A key feature of quantum mechanics which allows this in GBS is that binary "click" measurement operators are multimode projection operators, whose averages are probabilities. The positive- $P$ phase-space representation allows one to calculate these probabilities using efficient phase-space sampling methods, even though a direct sampling of the click distribution is exponentially hard. Since methods used to detect eavesdroppers involve measurement of moments or probabilities, this also suggests relevance to cryptographic steganography, in which messages could be hidden in the random outputs. We further demonstrate, using simulations in a different phase-space, the Wigner representation, how to certify the genuine $M$-partite entanglement of all $M=100$ nodes of a Gaussian network.

\section{GAUSSIAN BOSONIC NETWORKS}

We consider an $M$ mode bosonic network, with squeezed-state inputs to $N$ out of $M$ modes. A linear, unitary transformation is made to a set of $M$ output modes, combined with decoherence and losses. Measurements are carried out on the output state $\hat{\rho}^{\text {out }}$. The theoretical problem is to calculate quadrature correlations and binned counts for the output quantum state. To solve this, we utilize discrete Fourier transform methods for ensemble averages of grouped photon counts [33.

A squeezed-state is a minimum uncertainty state in which one of the input mode quadratures has its fluctuations reduced below the vacuum noise level [38 40]. We suppose that the squeezing of $\hat{\rho}^{\text {in }}$ for each excited mode is in the imaginary part of $\boldsymbol{\alpha}$. If each input is independent, the quantum state can be factorized into a product of single-mode states. Defining input quadrature operators $\hat{x}_{j}^{\text {in }}=\hat{a}_{j}^{\text {in }}+\hat{a}_{j}^{\text {in } \dagger}, \hat{p}_{j}^{\text {in }}=\left(\hat{a}_{j}^{\text {in }}-\hat{a}_{j}^{\text {in } \dagger}\right) / i$, so that $\left[\hat{x}_{\ell}^{\text {in }}, \hat{p}_{j}^{\text {in }}\right]=2 i \delta_{\ell j}$, the quantum inputs are defined by a squeezing vector $\boldsymbol{r}=\left[r_{1, \ldots} r_{N}\right]$.

The variances in each mode are:

$$
\begin{aligned}
& \left\langle\left(\Delta \hat{x}_{j}^{\text {in }}\right)^{2}\right\rangle=1+\gamma_{j}=e^{2 r_{j}} \\
& \left\langle\left(\Delta \hat{p}_{j}^{\text {in }}\right)^{2}\right\rangle=\left(1+\gamma_{j}\right)^{-1}=e^{-2 r_{j}} .
\end{aligned}
$$

The input photon numbers are $n_{j}=\sinh ^{2}\left(r_{j}\right)$, with coherences of $m_{j}=\left\langle\hat{a}_{j}^{2}\right\rangle=\sinh \left(r_{j}\right) \cosh \left(r_{j}\right)$. Pulsed squeezing [41] involves multiple longitudinal modes, with mismatches in time or frequency [23], as well as phase noise [42]. We model this experimental decoherence by an intensity transmissivity $T=1-\epsilon$ into the network, combined with a thermal input of $n_{j}^{t h}=\epsilon n_{j}$ uncorrelated photons per mode. Our model is similar to thermal squeezing [4], except with an invariant photon number.

The overall result is that the photon number is unchanged, and the coherence of each mode is reduced so that $\left\langle\hat{a}_{j}^{2}\right\rangle=\tilde{m}=(1-\epsilon) m\left(r_{j}\right)$.

\section{A. Phase-space representations}

At large $M$, number state expansions of the input state require exponentially many expansion coefficients to treat this. Instead, we use phase-space expansions 444 which allow a probabilistic representation of the input states. Two common phase-space approaches are used: the Wigner representation 45, and the generalized $P$-representation [46]. These methods do not assume Gaussianity, and applications to non-Gaussian photonic networks were already demonstrated [33, 47.

\section{Positive P-representation}

$P$-representations are normally ordered and therefore do not have any vacuum noise, making them efficient for simulating photo-detection measurements. The most suitable technique for non-classical photon-counting measurements is the generalized $P$-representation [46, 48, which has been applied to other large-scale bosonic simulations [49].

In this representation, $\hat{\rho}^{\text {in }}$ is expanded over a subspace of the complex plane defined by

$$
\hat{\rho}^{\text {in }}=R e \iint P(\boldsymbol{\alpha}, \boldsymbol{\beta}) \hat{\Lambda}(\boldsymbol{\alpha}, \boldsymbol{\beta}) d \mu(\boldsymbol{\alpha}, \boldsymbol{\beta}) .
$$

The operator basis $\hat{\Lambda}$ is a off-diagonal coherent state projector onto multimode Glauber [50] coherent states, and $d \mu(\boldsymbol{\alpha}, \boldsymbol{\beta})$ is an integration measure on the $2 M$ dimensional complex space of amplitudes $\boldsymbol{\alpha}, \boldsymbol{\beta}$, which in some cases reduce to simple real amplitudes.

For a squeezed input state $\hat{\rho}^{\text {in }}$, one obtains a positive P-distribution on a real subspace with $(\boldsymbol{\alpha}, \boldsymbol{\beta})=(\boldsymbol{x}, \boldsymbol{y})$, $d \mu(\boldsymbol{\alpha}, \boldsymbol{\beta})=d \boldsymbol{x} d \boldsymbol{y}$. If the input is $\hat{\rho}_{1 S} \equiv \prod_{j}\left|r_{j}\right\rangle\left\langle r_{j}\right|$ , a product of single-mode squeezed state density matrices, the solution for a squeezed state, based on onedimensional coherent state expansions [51] is:

$$
P(\boldsymbol{x}, \boldsymbol{y})=\prod_{j} C_{j} e^{-\left(x_{j}^{2}+y_{j}^{2}\right)\left(\gamma_{j}^{-1}+1 / 2\right)+x_{j} y_{j}},
$$

where the normalization constant is $C_{j}=$ $\sqrt{1+\gamma_{j}} /\left(\pi \gamma_{j}\right)$. 
In this approach, normally ordered operator moments are equivalent to stochastic moments [46], so that $\left\langle\hat{a}_{j_{1}}^{\dagger} \ldots \hat{a}_{j_{n}}\right\rangle=\lim _{S \rightarrow \infty}\left\langle\beta_{j_{1}} \ldots \alpha_{j_{n}}\right\rangle_{P}$, with quantum expectation values denoted \langle\rangle , and probabilistic averages with $S$ samples denoted \langle\rangle$_{P}$.

To create input samples for a squeezed state distribution $P(\boldsymbol{\alpha}, \boldsymbol{\beta})$, one uses real Gaussian noises with $\left\langle w_{i} w_{j}\right\rangle_{P}=\delta_{i j}$, to generate random phase-space samples $\vec{\alpha}=[\boldsymbol{\alpha}, \boldsymbol{\beta}]=\left[\alpha_{1}, \ldots \alpha_{2 M}\right]$. The stochastic model for a pure or thermalized squeezed state, $[\boldsymbol{\alpha}, \boldsymbol{\beta}]$, is given by [2]:

$$
\begin{aligned}
\alpha_{j} & =\delta_{j+} w_{j}+i \delta_{j-} w_{j+M} \\
\beta_{j} & =\delta_{j+} w_{j}-i \delta_{j-} w_{j+M} .
\end{aligned}
$$

The coefficients $\delta_{j \pm}$ must satisfy $\delta_{j \pm}=\sqrt{\left(n_{j} \pm \tilde{m}_{j}\right) / 2}$, which gives real amplitudes for $n_{j} \leq \tilde{m}_{j}$, and complex amplitudes for $n_{j}>\tilde{m}_{j}$.

\section{Wigner representation}

Other possible phase-space methods include the Wigner [45, 52] and Q-function [53], methods with symmetric and anti-normal ordering respectively. These give exponentially larger sampling errors [42] for intensity correlation due to their extra vacuum noise, while the Glauber P-representation is singular for squeezed states. These methods have a classical phase-space, in which $\beta_{j}=\alpha_{j}^{*}$. The Wigner representation is best for analyzing multipartite entanglement, with data coming from quadrature measurements [54 56, which have been carried out at an increasingly large scale [17-19, 57, 58.

For an input quantum density matrix $\hat{\rho}^{\text {in }}$ the Wigner distribution $W(\boldsymbol{\alpha})$ is written most compactly as [44, 59, 60]:

$$
W(\boldsymbol{\alpha})=\frac{1}{\pi^{2 N}} \int d^{2} \mathbf{z} \operatorname{Tr}\left[\hat{\rho}^{\mathrm{in}} e^{i \mathbf{z} \cdot(\hat{\mathbf{a}}-\boldsymbol{\alpha})+i \mathbf{z}^{*} \cdot\left(\hat{\mathbf{a}}^{\dagger}-\boldsymbol{\alpha}^{*}\right)}\right]
$$

More generally, $\sigma$-ordered classical bosonic representations [61] are defined using an $\sigma$ parameter signifying the relative amount of vacuum noise, with $\sigma=0$ for normal ordering or P-representations and $\sigma=1 / 2$ for symmetric ordering or Wigner representations. For pure and thermalized squeezed state inputs [42, 62, the $\sigma$-ordered classical phase-space stochastic amplitude is

$$
\alpha_{j}=\delta_{j+} w_{j}+i \delta_{j-} w_{j+M},
$$

where $\delta_{j \pm}$ has the requirement that:

$$
\delta_{j \pm}=\sqrt{\left(n_{j}+\sigma \pm \tilde{m}_{j}\right) / 2} .
$$

\section{B. Network Transmission}

Once a set of input states is simulated, it can be transformed and used to sample the output state in any of these representations. An input density matrix $\hat{\rho}^{\text {in }}$ is changed by a linear photonic network to an output density matrix $\hat{\rho}^{\text {out }}$. For unitary transformations $\boldsymbol{T}$, the phase-space amplitudes are transformed deterministically, where $\boldsymbol{\alpha}^{\prime}=\boldsymbol{T} \boldsymbol{\alpha}, \boldsymbol{\beta}^{\prime}=\boldsymbol{T}^{*} \boldsymbol{\beta}$, in all representations. In the generalized P-representation one may include a non-unitary transmission matrix to take account of losses, which is equivalent to a master equation [63.

For normal ordering in a linear network, the output density matrix has a simple form [64, including linear couplings and losses:

$$
\hat{\rho}^{\text {out }}=\Re \iint P(\boldsymbol{\alpha}, \boldsymbol{\beta}) \hat{\Lambda}\left(\boldsymbol{T} \boldsymbol{\alpha}, \boldsymbol{T}^{*} \boldsymbol{\beta}\right) \mathrm{d} \mu(\boldsymbol{\alpha}, \boldsymbol{\beta}) .
$$

The input distribution $P(\boldsymbol{\alpha}, \boldsymbol{\beta})$ may no longer be restricted to the real axes if there are input thermal photons included to model decoherence with $\boldsymbol{n}^{\text {th }} \neq 0$.

For other types of ordering, vacuum noise must be included from the reservoirs that couple to the system modes, causing decoherence. This is achieved by noting that for a vacuum state, the input and output correlations are identical, and for $\sigma$-ordering, $\left\langle\beta_{i} \alpha_{j}\right\rangle=\left\langle\beta_{i}^{\prime} \alpha_{j}^{\prime}\right\rangle=$ $\sigma \delta_{i j}$. It is therefore necessary to add additional vacuum noise if $\sigma>0$ and $\boldsymbol{T}$ is non-unitary. This is achieved through defining an hermitian decoherence matrix, $\boldsymbol{D} \equiv$ $\boldsymbol{I}-\boldsymbol{T}^{\dagger} \boldsymbol{T}$, which has a decomposition $\boldsymbol{D} \equiv \boldsymbol{U} \boldsymbol{\lambda}^{2} \boldsymbol{U}^{\dagger}$, where $\boldsymbol{\lambda}$ is diagonal and positive. The matrix square root is $\boldsymbol{B}=\boldsymbol{U} \boldsymbol{\lambda} \boldsymbol{U}^{\dagger}$, and the output amplitudes are:

$$
\boldsymbol{\alpha}^{\prime}=\boldsymbol{T} \boldsymbol{\alpha}+\sqrt{\frac{\sigma}{2}} \boldsymbol{B}(\boldsymbol{u}+i \boldsymbol{v}) .
$$

This ensure that vacuum noise is unchanged.

\section{QUANTUM MEASUREMENTS}

We consider two type of measurements in detail, namely quadrature detection, and efficient photodetectors that saturate for more than one count.

\section{A. Phase-space representations of measurement operators}

\section{Quadrature detectors}

For quadrature detection, the quadrature phase amplitudes of each output mode are $\hat{x}_{i}^{\theta}=\hat{a}_{i} e^{-i \theta}+\hat{a}_{i}^{\dagger} e^{i \theta}$, with special cases, for $\theta_{i}=0, \pi / 2$ of $\hat{x}_{i}$ and $\hat{p}_{i}$, in a rotating frame. The measurable quadrature correlations are $\mathcal{C}(\boldsymbol{m}, \boldsymbol{\theta})$, where $\boldsymbol{m}=\left(m_{1}, \ldots m_{n}\right)$, and $\boldsymbol{\theta}=\left(\theta_{1}, \ldots \theta_{n}\right)$ :

$$
\mathcal{C}(\boldsymbol{m}, \boldsymbol{\theta})=\left\langle\prod_{j=1}^{n}\left[\hat{x}_{j}^{\theta_{j}}\right]^{m_{j}}\right\rangle .
$$

This is directly simulated in the Wigner representation. The phase-space variables are $x_{i}^{\theta}=\alpha_{i}^{\prime} e^{-i \theta}+\alpha_{i}^{\prime \dagger} e^{i \theta}$, and 
the correlations are calculated by replacing $\hat{x}_{j}^{\theta_{j}} \rightarrow x_{j}^{\theta_{j}}$, and averaging over the Wigner function.

Hence, one simply has to generate the input amplitudes according to $\mathrm{Eq} 2.6$, transform them according to $\mathrm{Eq}$ (2.9), and average over an ensemble of random events to obtain the output measured correlations. These are given a detailed analysis in Section $V$

\section{Photon number detectors}

For photon number resolving photodetectors, the output number operator is $\hat{n}_{j}^{\prime}=\hat{a}_{j}^{\dagger \text { †out }} \hat{a}_{j}^{\text {out }}$. The $n$-th order Glauber correlation function is [50]:

$$
G^{(n)}\left(c_{j}\right)=\left\langle: \prod_{j=1}^{M}\left(\hat{n}_{j}^{\prime}\right)^{c_{j}}:\right\rangle,
$$

where $c_{j}=0,1,2 \ldots \ldots$ is the number of counts at the $j$-th detector, and $n=\sum c_{j}$ is the total measurement order. The corresponding phase-space observable is obtained by replacing $\hat{n}_{j}^{\prime} \rightarrow n_{j}^{\prime}$, where $n_{j}^{\prime}=\alpha_{j}^{\prime} \beta_{j}^{\prime}$ is the output number variable, sampled with probability $P(\boldsymbol{\alpha}, \boldsymbol{\beta})$. To calculate the output number distribution, there are a number of methods [10], for transforming these correlations into the observed distributions, but in this paper we focus on the saturating, or on-off detector, as recent experiments use this type of measurement.

For saturating photodetectors, all non-zero counts give an identical output. The "on-off" click projection operator is 65:

$$
\hat{\pi}_{j}\left(c_{j}\right)=: e^{-\hat{n}_{j}^{\prime}}\left(e^{\hat{n}_{j}^{\prime}}-1\right)^{c_{j}}:
$$

where $c_{j}=0,1$ is the number of measured counts at the $j$-th detector, and the output number operator is $\hat{n}_{j}^{\prime}=\hat{a}_{j}^{\dagger \text { out }} \hat{a}_{j}^{\text {out }}$. Multi-mode results are given by an $M$ digit binary number $c$. This has $2^{M}$ possible patterns available. For a set $S$ of $M_{S}$ sites, each binary number $\mathbf{c}_{S}$ has an $M_{s}$-order correlation operator of $\hat{\Pi}_{S}\left(\mathbf{c}_{S}\right)=$ $\bigotimes_{j \in S} \hat{\pi}_{j}\left(c_{j}\right)$. The corresponding expectation,

$$
T\left(\mathbf{c}_{S}\right)=\left\langle\bigotimes_{j \in S} \hat{\pi}_{j}\left(c_{j}\right)\right\rangle,
$$

is the Torontonian function [11] for Gaussian inputs, if the set of sites corresponds to all $M$ output channels. As this is normally ordered, it has a direct correspondence with a phase-space function in the positive $\mathrm{P}$ representation. The phase-space observable is given by replacing $\hat{n}_{j}^{\prime} \rightarrow n_{j}^{\prime}$, where $n_{j}^{\prime}=\alpha_{j}^{\prime} \beta_{j}^{\prime}$ is the output number variable sampled with probability $P(\boldsymbol{\alpha}, \boldsymbol{\beta})$.

In all cases, the corresponding $M_{s}$-th order moment is simulated by replacing $\hat{\pi}_{j}$ with the randomly sampled complex number $\pi_{j}$. We note that the operators $\hat{\Pi}_{S}\left(\mathbf{c}_{S}\right)$ are projectors. As a result, their expectations are the probabilities [66] of measuring the count pattern $\mathbf{c}_{S}$.

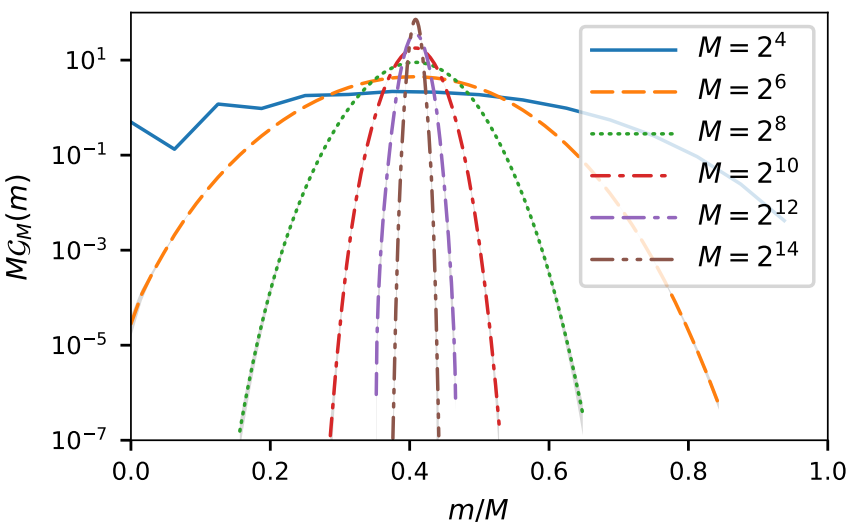

Figure 1. Theoretical scaling of total grouped count distribution with $M$. Results are for $M_{\mathcal{G}}^{(M)}(m)$ vs $m / M$, for $\boldsymbol{r}=1, \epsilon=0, N=M / 2$ and random unitaries. Mode numbers were $M=2^{4}, 2^{6}, 2^{8}, 2^{10}, 2^{12}, 2^{14}$, with sample numbers of $10^{8}, 10^{6}, 10^{5}, 1.6 \times 10^{4}, 4 \times 10^{3}, 2 \times 10^{3}$ respectively. The transmission matrices are random unitaries.

Since there are exponentially many possible count patterns c, the probability of measuring any individual pattern, $\left\langle\hat{\Pi}_{S}\left(\mathbf{c}_{S}\right)\right\rangle$, becomes infinitesimal for large $M_{s}$. A direct measurement cannot obtain all such correlations in less than exponential time. Thus, it is hard to calculate all probabilities, and it is also hard to measure them [33, 47.

\section{Grouped or marginal probabilities}

Grouped counts are therefore essential for verifying GBS statistics at large $M$, in order to obtain measurable probabilities. One must simulate and measure the $n$-th order grouped probabilities, $\mathcal{G}_{\boldsymbol{S}}^{(n)}(\boldsymbol{m})$, where $n=\sum_{j=1}^{d} M_{j} \leq M$, is the total probability order [50], and:

$$
\mathcal{G}_{S}^{(n)}(\boldsymbol{m})=\left\langle\prod_{j=1}^{d}\left[\sum_{\sum c_{i}=m_{j}} \hat{\Pi}_{S_{j}}(\boldsymbol{c})\right]\right\rangle .
$$

These are the $d$-dimensional grouped count probabilities of observing $\boldsymbol{m}=\left(m_{1} \ldots m_{d}\right)$ grouped counts in disjoint sets $\boldsymbol{S}=\left(S_{1}, S_{2}, \ldots\right)$ of $\boldsymbol{M}=\left(M_{1}, M_{2}, \ldots\right)$ output modes. If $n<M$, they include low-order marginal probabilities often proposed for verification purposes, with $M-n$ outputs ignored. The first-order correlation with $n=1, S=\{j\}$ is the count probability in the $j$-th channel. Similarly, $n=M$ and $\boldsymbol{S}=(\{1\},\{2\}, \ldots)$ gives the Torontonian. For sequential channel groups, the sets $\boldsymbol{S}$ are simply denoted by their sizes $\boldsymbol{M}$. Using this notation, $\mathcal{G}_{M}^{(M)}(m) \equiv \mathcal{G}_{\{1,2, \ldots M\}}^{(M)}(m)$ is the probability for observing $m$ clicks in any pattern, as reported in recent experiments 23 . 
We use the terminology of quantum optics [50] to define the measurement order, $n$, since the binned correlations involve simultaneous measurements at $n$ different sites. However, the $\mathcal{G}_{\boldsymbol{S}}^{(n)}(\boldsymbol{m})$ are probabilities obtained from some or all of the detectors. One can extract moments from the resulting distributions, and the resulting statistical moments can also have various orders from $1, \ldots n$. It is important to distinguish the original measurement order which depends on the number of modes, from the statistical moment orders that are extracted later.

Calculating these quantities appears intractable at first: how can one compute the sum of exponentially many terms, if each is exponentially hard? Yet, highorder correlations are readily simulated on replacing the operator $\hat{\pi}_{i}$ by the phase-space variable $\pi_{i}$, and averaging over the probability $P(\boldsymbol{\alpha}, \boldsymbol{\beta})$. The summation over grouped correlations is achieved through defining angles $\theta_{j}=2 \pi /\left(M_{j}+1\right)$, with a Fourier observable $\tilde{\mathcal{G}}$ defined for $k_{j}=0, \ldots M_{j}$, where $j=1, \ldots d$. The grouped probability is then obtained from a $d$-dimensional inverse discrete Fourier transform, so that:

$$
\begin{aligned}
\tilde{\mathcal{G}}_{S}^{(n)}(\boldsymbol{k}) & =\left\langle\prod_{j=1}^{d} \bigotimes_{i \in S_{j}}\left(\pi_{i}(0)+\pi_{i}(1) e^{-i k_{j} \theta_{j}}\right)\right\rangle_{P} \\
\mathcal{G}_{\boldsymbol{S}}^{(n)}(\boldsymbol{m}) & =\frac{1}{\prod_{j}\left(M_{j}+1\right)} \sum_{\boldsymbol{k}} \tilde{\mathcal{G}}_{\boldsymbol{S}}^{(n)}(\boldsymbol{k}) e^{i \sum k_{j} \theta_{j} m_{j}}
\end{aligned}
$$

All combinations of terms vanish in the inverse Fourier transform except those terms with $\boldsymbol{m}$ counts. This algorithmic procedure is highly scalable. To demonstrate this, two simulation codes were written and tested. Exact Torontonians were simulated for small networks. Analytically tractable inputs were used to test large networks. Excellent agreement was found in all cases.

To demonstrate this technique for quantum squeezed inputs, we graph the grouped count probability in Fig (1) for sizes up to $M=16,000$, using squeezed states with $r=1, \epsilon=0$, for $N=M / 2$ inputs, and random unitaries.

\section{GROUPED COUNT VERIFICATION IN GBS EXPERIMENTS}

The grouped probabilities provide a signature of a quantum state. Clearly, they must be measurable and have a low sampling error. To validate results, the theoretical sampling error $E_{t}$ must be less than the experimental sampling error $E_{e}$, where the experimental sampling error depends on the number of samples used, and scales as $E_{e} \propto c_{e} S_{e}^{-1 / 2}$. Experiment and simulations have similar time-scales for comparable error-bars.

Due to internal averaging, single group measures are less sensitive to the unitary as $n$ increases, but are very sensitive to decoherence. Count "fingerprints" with more groups are also needed for a complete test, and one is computed below. Many such measures are available, both from experimental data and from simulations.

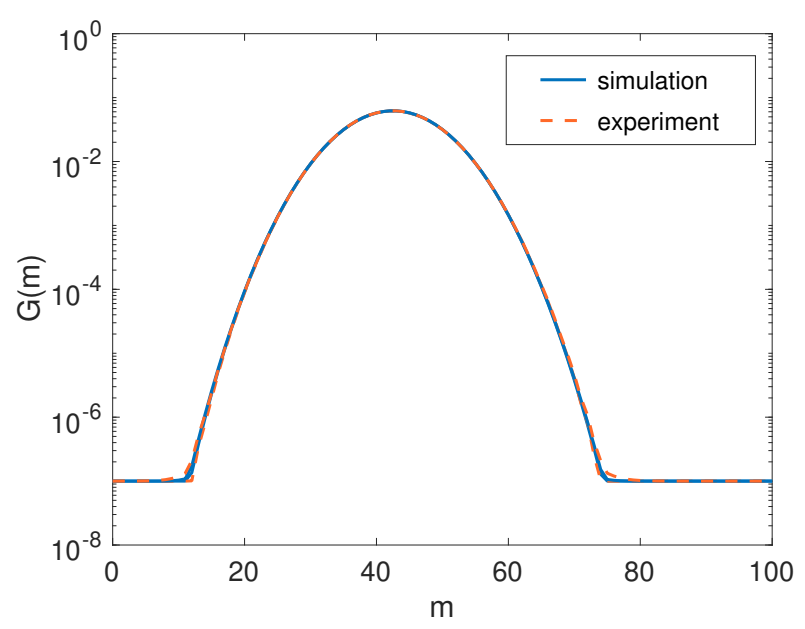

Figure 2. Comparison of theory with experiment of $\mathcal{G}_{100}^{(100)}(m)$, for a 100 channel GBS total count distribution. Solid blue line is the theoretical prediction with $\epsilon=0.0932$ relative decoherence and $1.2 \times 10^{6}$ samples. The orange dashed line is the experimental data obtained from $5 \times 10^{7}$ samples.

\section{A. Comparisons with a GBS experiment}

To compare theory to experiment, squeezing vectors and transmission data from a recent 100-mode Gaussian boson sampling experiment were obtained [23] and simulated with $1.2 \times 10^{6}$ samples. The data was a 50 mode vector of amplitudes $\boldsymbol{r}$, a $50 \times 100$ transmission matrix $\boldsymbol{T}$, and $5 \times 10^{7}$ measured click patterns. The experimental data was used to calculate grouped correlations, and compared to simulations with a standard chi-squared test [67, using a lower cut-off of 10 counts per bin [36, 68. Statistical methods and tests of the codes are given in the Appendix.

For $k$ significant bins, one expects $\chi_{c}^{2} / k \approx 1$. Simulating total counts, $\mathcal{G}_{M}^{(M)}(m)$, with pure squeezed-state inputs gave a large chi-squared value of $\chi_{c}^{2} / k=9.5 \times 10^{3} \gg$ 1 , with $k=63$ valid data points. Additionally, we tested a 100 mode fully thermalized model. This gave an even larger discrepancy. The chi-squared value was $\chi_{t h}^{2} / k=6.1 \times 10^{4} \gg 1$, confirming a prediction [69] that one can distinguish boson sampling from uniform distributions.

Better agreement with experiment was obtained with a small admixture of thermal inputs. For optimal fitting, we included an $\epsilon=0.0932 \pm 0.0005$ thermal component to model longitudinal mode mismatching. Transmission amplitudes were multiplied by $1.0235 \pm 0.0005$. Results of simulations are given in Fig (2). This agrees with experiment over a range of six orders of magnitude in the measurable grouped probabilities. A chi-squared value of $\chi_{\epsilon}^{2} / k=6.5 \pm 1$ was obtained, giving three orders of magnitude lower values than with pure state inputs. Residual discrepancies may be from nonlinearities.

Fig (3) shows $\mathcal{G}_{50,50}^{(100)}\left(m_{1}, m_{2}\right)$, which is a two- 


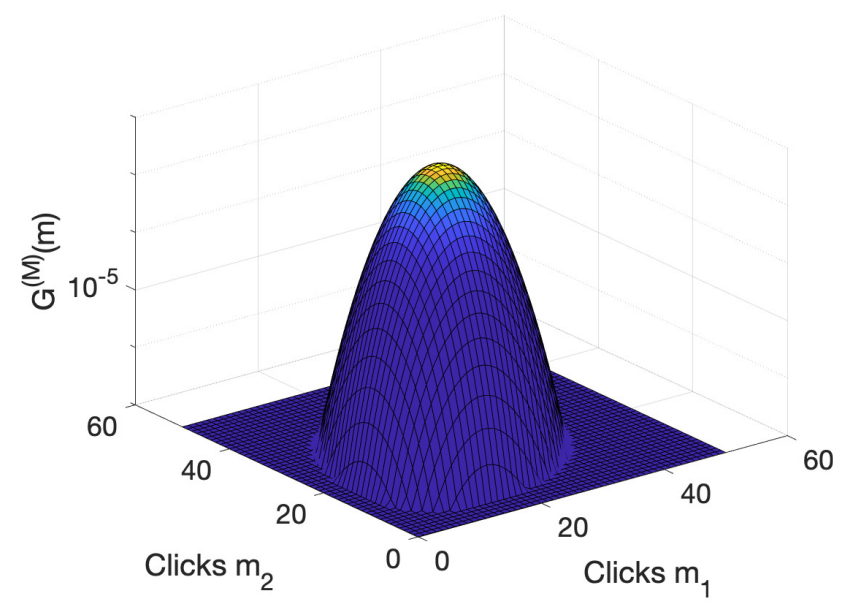

Figure 3. Simulation of a 100 channel GBS count distribution binned into $d=2$ dimensions, $\mathcal{G}_{50,50}^{(100)}\left(m_{1}, m_{2}\right)$, with $1.2 \times 10^{6}$ samples. There are $51^{2}$ data points in the distribution, leading to over 1000 distinct data-points. Including decoherence, the differences between theory and experiment are negligible on this scale.

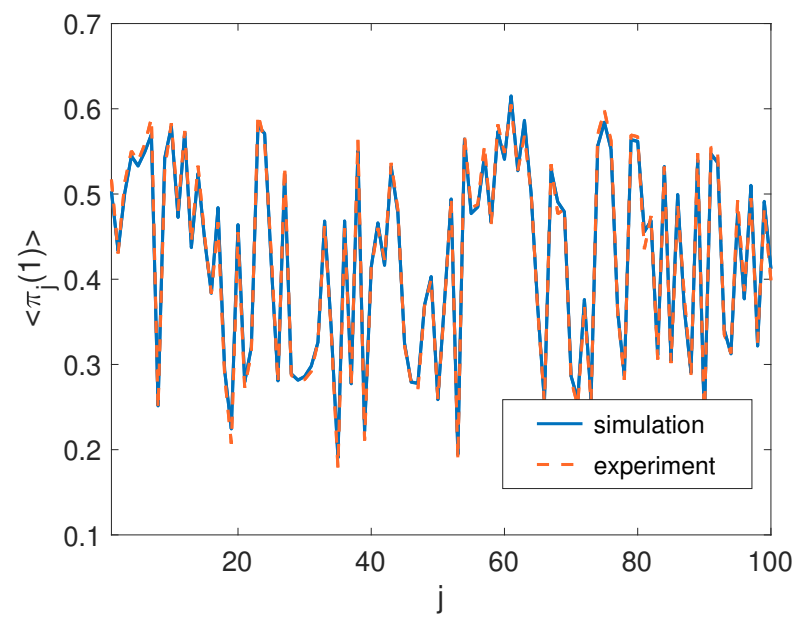

Figure 4. Comparison of theory vs experiment for a 100 channel GBS count probability per channel, $\mathcal{G}_{\{j\}}^{(1)}(1) \equiv\left\langle\hat{\pi}_{j}(1)\right\rangle$, versus mode $j$. Blue line is the theory with with $\epsilon=0.0932$ added decoherence, orange dashed line is obtained from $5 \times 10^{7}$ experimental data records. Computational sampling errors with $1.2 \times 10^{6}$ samples were negligible on this scale.

dimensional binning of the 100-th order probabilities. Any number of bins - up to $M$ - are feasible in principle. However, experimental sampling errors increase as the grouping dimension increases, giving a limit of $d=6$ dimensions with currently available experimental data. As another comparison, the marginal count probability per channel $\mathcal{G}_{\{j\}}^{(1)}(1)=\left\langle\pi_{j}(1)\right\rangle$, is graphed in Fig 4 . This also shows good agreement with experiment.

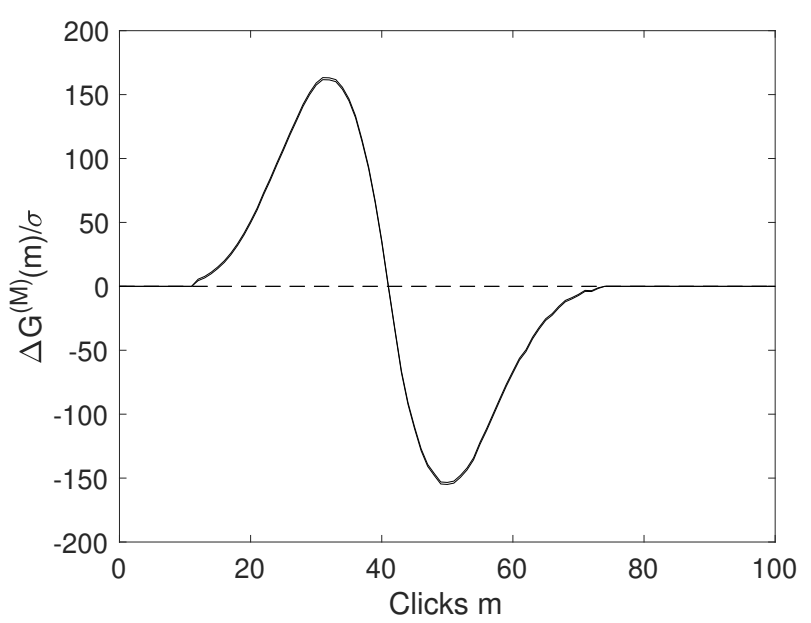

Figure 5. Normalized difference of simulation versus experimental count distribution, excluding decoherence. Results are for $\Delta \mathcal{G}_{M}^{(M)}(m) / \sigma_{m}$ vs $m$, with sample numbers of $1.2 \times 10^{6}$. The error bars indicate errors due to finite experimental counts plus theoretical sampling errors. The results are cut off for all counts less than 10 .

\section{B. Detailed statistical comparisons}

We now consider the details of the comparisons and the inferred decoherence from the grouped 100-th order correlations in a GBS experiment, as compared to a phasespace simulation. We wish to compare two hypotheses. The first, $\mathcal{H}_{0}$, is that the correlations are given by the experimental squeezing and transmission matrices. The second, $\mathcal{H}_{1}$, is that there is additional decoherence, modeled by an thermal fraction $\epsilon$, with an unchanged photon number.

In both cases, the experimental counts are the same. However, graphing raw experimental and theoretical count probabilities is not useful for comparative purposes, as the probabilities appear nearly identical to the naked eye 67]. Due to the accuracy of the data, with over $10^{7}$ total counts, it is much more useful to graph the normalized deviation between theoretical and experimental probabilities, as described in the Appendix:

$$
z_{m}=\frac{\Delta \mathcal{G}_{M}^{(M)}(m)}{\sigma_{m}}=\frac{\mathcal{G}(m)-\mathcal{G}^{e}(m)}{\sigma_{m}} .
$$

For good agreement between theory and experiment, one expects a normalized difference of unity. Figure (5), shows the normalized discrepancy between theory and experiment in $\mathcal{G}_{M}^{(M)}(m)$, in a simulation having no decoherence. An inspection of the graph shows very significant differences between the theoretical and experimental count probabilities, with $|z| \gg 1$. This is reflected in the $\chi^{2}$ results for the null hypothesis $\mathcal{H}_{0}$, where one obtains an extremely large value of $5.9 \times 10^{5}$, out of 63 valid data points having more than 10 counts. This gives a discrepancy ratio of $\chi_{c}^{2} / k=9.5 \times 10^{3} \gg 1$. 


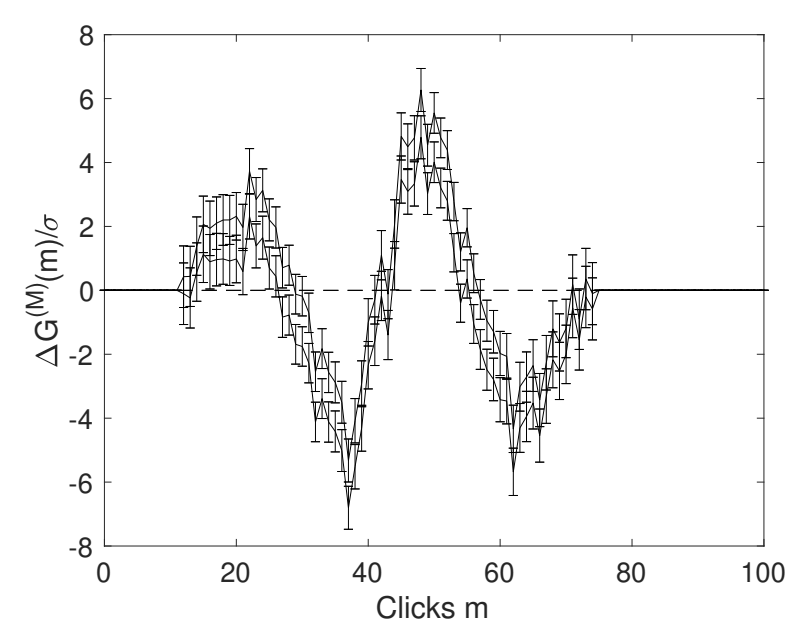

Figure 6. Normalized difference of simulation versus experimental count distribution, including decoherence. Results are for $\Delta \mathcal{G}_{M}^{(M)}(m) / \sigma_{m}$ vs $m$, with sample numbers of $1.2 \times 10^{6}$. The error bars indicate RMS errors due to finite experimental counts, plus theoretical sampling errors (which are $50 \%$ smaller). Results are cut off for counts less than 10, where the count data is less reliable. The maximum error is reduced by about two orders of magnitude compared to the coherent model.

Clearly, when decoherence is excluded, the experiment strongly disagrees with a simple, coherent GBS model. Therefore, the hypothesis of no decoherence, apart from losses, has a vanishingly small probability. The hypothesis of a fully thermal model with $\epsilon=1$ is less likely still. With this model, the total discrepancy ratio is $\chi_{t h}^{2} / k=6.1 \times 10^{4} \gg 1$. Hence, the output is easily distinguishable from a thermal one 69].

Figure 6, , shows the differences in $\mathcal{G}_{M}^{(M)}(m)$, between experimental and simulated probabilities with a small decoherence of $\epsilon=0.0932$, as described above. The transmission amplitude was increased by a factor of 1.0235 to improve the fit. This is a small correction, since even small deviations can result in large chi-squares. The new graph shows much smaller differences between the theoretical and experimental count probabilities, with $|z| \lesssim 5$.

For the hypothesis $\mathcal{H}_{1}$, with additional decoherence, the $\chi_{\epsilon}^{2}$ value is $400 \pm 50$. The total ratio is $\chi_{\epsilon}^{2} / k=$ $6.5 \pm 1 \sim O(1)$, more than 1000 times smaller than for a pure state. This indicates that the hypothesis of additional decoherence is more compatible with experimental measurements. These results show good agreement with a model of GBS including a small thermal decoherence. Other physical effects including nonlinearities may explain the remaining discrepancies.

The phase-space errors here were $50 \%$ less than the experimental errors, and could be reduced further, at the cost of longer computation time. There were 40 times more experimental than theoretical samples. Hence, this simulation is comparable or better than experimental efficiency. The error ratio depends on the observations.
The same technique is applicable to any measurable distribution, including lower order marginal probabilities and multiple partitions. We plotted an example of a two-dimensional grouped probability distribution in the previous subsection. This has similar properties, with a distribution close to the experimental one, and includes thousands of data points.

In general it is possible to check the GBS hypothesis for any set of parameters and marginal or grouped probability distributions. Measurable, grouped probability distributions of GBS experimental data can be simulated to high accuracy. We have simulated correlation orders from first up to 16,000 th order. The computational time depends on the complexity of extraction of the binned correlations, and on the error requirements.

There are many correlation tests possible. Thus, to disprove a classical mock-up, one could simply use a large, randomly chosen subset of tests of all orders. Just as with other RNG tests, it is increasingly unlikely that a range of statistical tests like this can be faked. We conjecture that the multidimensional grouped probabilities, as in Fig 3, have the most potential for this due to their polynomially large number of probability samples.

\section{N-PARTITE ENTANGLEMENT}

These experiments typically lead to entangled outputs. However, the entanglement is demonstrated most directly using a different type of measurement. We will illustrate this for one type of $M$-partite entangled state that is generated from one or two squeezed vacuum states. In this section, we briefly outline the known method for generating such a state [54, 56]. In short, the squeezed inputs are first combined across a single beam splitter to create a two-mode Einstein-Podolsky-Rosen (EPR) entangled state [70]. One of the outputs is then passed through $M-2$ beam splitters [54, 56].

\section{A. Multi-mode entanglement theory}

The overall set-up has $M$ inputs $\hat{a}_{j}^{\text {in }}$, where the first two inputs are orthogonally squeezed vacuum states. In particular, $\hat{a}_{2}^{\text {in }}$ is a squeezed vacuum input with $\Delta^{2} \hat{x}_{2}^{\text {in }}=$ $e^{-2 r}$, and $\hat{a}_{1}^{\text {in }}$ is squeezed vacuum input with $\Delta^{2} \hat{p}_{1}^{\text {in }}=$ $e^{-2 r}$. Here $r>0$ is the squeezing parameter. All other inputs are vacuum states, implying $\Delta^{2} \hat{x}_{j}^{\text {in }}=\Delta^{2} \hat{p}_{j}^{\text {in }}$. The inputs are combined across a total of $M-1$ beam splitters. We use the notation $\Delta^{2} \hat{x}$ to mean the variance of $\hat{x}$ i.e. $\Delta^{2} \hat{x}=(\Delta \hat{x})^{2}=\left\langle\hat{x}^{2}\right\rangle-\langle\hat{x}\rangle^{2}$.

To create two-mode EPR entanglement, inputs 1 and 2 are passed through beam splitter $B S 1$, with reflectivity $R_{1}^{2}$ and $T_{1}^{2}=1-R_{1}^{2}$, according to

$$
\begin{aligned}
& \hat{a}_{1}^{(1)}=R_{1} \hat{a}_{1}^{\text {in }}+T_{1} \hat{a}_{2}^{\text {in }} \\
& \hat{a}_{2}^{(1)}=T_{1} \hat{a}_{1}^{\text {in }}-R_{1} \hat{a}_{2}^{\text {in }} .
\end{aligned}
$$


The output of $\hat{a}_{1}$ is $\hat{a}_{1}^{(1)}$. It is straightforward to show using the approach developed in [70] that the two outputs are EPR correlated with respect to the quadrature phase amplitudes, i.e.

$$
\begin{aligned}
\Delta^{2}\left(\hat{x}_{1}-\hat{x}_{2}^{(1)}\right) & =2 e^{-2 r} \\
\Delta^{2}\left(\hat{p}_{1}+\hat{p}_{2}^{(1)}\right) & =2 e^{-2 r} .
\end{aligned}
$$

More details are given in [71, 72, where EPR steering is also considered. For large $r$, both variances become zero. EPR entanglement can also be created from one squeezed input $\hat{a}_{1}^{\text {in }}$ to give

$$
\begin{aligned}
\Delta^{2}\left(\hat{x}_{1}-\hat{x}_{2}^{(1)}\right) & =2 \\
\Delta^{2}\left(\hat{p}_{1}+\hat{p}_{2}^{(1)}\right) & =2 e^{-2 r} .
\end{aligned}
$$

To generate multipartite entanglement, the field $\hat{a}_{2}^{(1)}$ is combined across a second $B S 2$ with reflectivity $R_{2}^{2}$ and $T_{2}^{2}=1-R_{2}^{2}$, according to 54

$$
\begin{aligned}
& \hat{a}_{2}^{(2)}=R_{2} \hat{a}_{2}^{(1)}+T_{2} \hat{a}_{3}^{\text {in }} \\
& \hat{a}_{3}^{(2)}=T_{2} \hat{a}_{2}^{(1)}-R_{2} \hat{a}_{3}^{\text {in }} .
\end{aligned}
$$

The output of field $\hat{a}_{2}$ is $\hat{a}_{2}^{(2)}$. For $M=3$, there are only two beam splitters, and the output of $\hat{a}_{3}$ is $\hat{a}_{3}^{(2)}$. For $M=4$, the process continues with another beam splitter

$$
\begin{aligned}
& \hat{a}_{3}^{(3)}=\left(R_{3} \hat{a}_{3}^{(2)}+T_{3} \hat{a}_{4}^{\text {in }}\right) \\
& \hat{a}_{4}^{(3)}=\left(T_{3} \hat{a}_{3}^{(2)}-R_{3} \hat{a}_{4}^{\text {in }}\right) .
\end{aligned}
$$

The output of mode $\hat{a}_{3}$ is $\hat{a}_{3}^{(3)}$ and the output of mode $\hat{a}_{4}$ is $\hat{a}_{4}^{(3)}$.

It is possible to continue in this way, and to select the reflectivities of a string of beam splitters so that we obtain, from two squeezed inputs, the following solution for the final outputs $\hat{a}_{i}^{\text {out }}$, given by $\hat{a}_{i}^{\text {out }}={\hat{a_{i}}}^{(i)}, i=$ $1, . ., M-1$ and $\hat{a}_{M}^{\text {out }}=\hat{a}_{i}{ }^{(i-1)}$ :

$$
\begin{aligned}
& \xi_{x}=\Delta^{2}\left(\hat{x}_{1}^{\text {out }}-\frac{1}{\sqrt{M-1}} \sum_{i>1}^{M} \hat{x}_{i}^{\text {out }}\right)=2 e^{-2 r} \\
& \xi_{p}=\Delta^{2}\left(\hat{p}_{1}^{\text {out }}+\frac{1}{\sqrt{M-1}} \sum_{i>1}^{M} \hat{p}_{i}^{\text {out }}\right)=2 e^{-2 r} .
\end{aligned}
$$

To achieve this, the reflectivity $R_{k}^{2}$ for $k$-th beam splitter, where $k=1, . ., M-1$, is $R_{M-1}^{2}=1 / 2, R_{M-2}^{2}=\frac{1}{3}$, $R_{M-k}^{2}=1 /(k+1)$ for $k<M-1$, with $R_{1}^{2}=1 / 2$. This is explained in more detail in [54, 56].

\section{B. Unitary matrix}

The corresponding unitary matrix for the set-up is obtained by first introducing a vector of reflection ampli- tudes, defined by

$$
\begin{aligned}
R_{j} & =\sqrt{\frac{1}{M-j+1}}, \quad 1<j \leq M \\
R_{1} & =\sqrt{\frac{1}{2}} \\
T_{j} & =\sqrt{1-R_{j}^{2}} .
\end{aligned}
$$

We express the transformation of the $M$ input modes into $M$ genuinely entangled output modes as a unitary matrix $U$. The output modes are

$$
\left(\begin{array}{c}
\hat{a}_{1}^{\text {out }} \\
\\
\hat{a}_{k}^{\text {out }} \\
\cdot \cdot \\
\hat{a}_{M}^{\text {out }}
\end{array}\right)=U\left(\begin{array}{c}
\hat{a}_{1}^{\text {in }} \\
\\
\\
\hat{a}_{k}^{\text {in }} \\
\cdot \cdot \\
\hat{a}_{M}^{\text {in }}
\end{array}\right) .
$$

Defining $R_{0}=-1$ and $R_{M}=1$, the elements $U_{k j}$ of the $M \times M$ unitary $U$ matrix for $j, k=1, . . M$ are given by:

$$
\left\{\begin{array}{ll}
U_{k j}=0 & j>k+1 \\
U_{k j}=R_{1} & j=k=1 \\
U_{k k}=-R_{k} R_{k-1} & k<M \\
U_{k(k+1)}=T_{k} & 1 \leq j<k+1 \\
U_{k j}=-R_{k} T_{k-1} . . T_{j} R_{j-1} & 1
\end{array} .\right.
$$

\section{N-partite entanglement simulations}

We now consider how to use phase-space simulations to model an entangled bosonic network, such as the one described in the last section. This result can be readily simulated, and we find that one can verify genuine $M$ partite entanglement [54, 56], where we take $M=100$. The optimal simulation method is the Wigner representation, which for quadrature measurement is the natural approach, requiring no ordering corrections. Other methods gave larger sampling errors. As one might guess intuitively, it is optimal to use the representation that matches the measurement operator [73].

This case demonstrates the high efficiency of Wigner phase-space methods for simulating quadrature measurements, although it is a completely different type of measurement to the click detection often used in GBS.

We follow the definitions given in Eq. (5.6). Let $\hat{u}=$ $\hat{x}_{1}-\frac{1}{\sqrt{M-1}}\left(\hat{x}_{2}+\hat{x}_{3}+. . \hat{x}_{M}\right)$ and $\hat{v}=\hat{p}_{1}+\frac{1}{\sqrt{M-1}}\left(\hat{p}_{2}+\hat{p}_{3}+\right.$ ... $\left.\hat{p}_{M}\right)$, then the observation of

$$
(\Delta \hat{u})(\Delta \hat{v})<\frac{2}{(M-1)}
$$

confirms $M$-partite entanglement for all $M$. The observation of

$$
(\Delta \hat{u})^{2}+(\Delta \hat{v})^{2}<\frac{4}{M-1}
$$




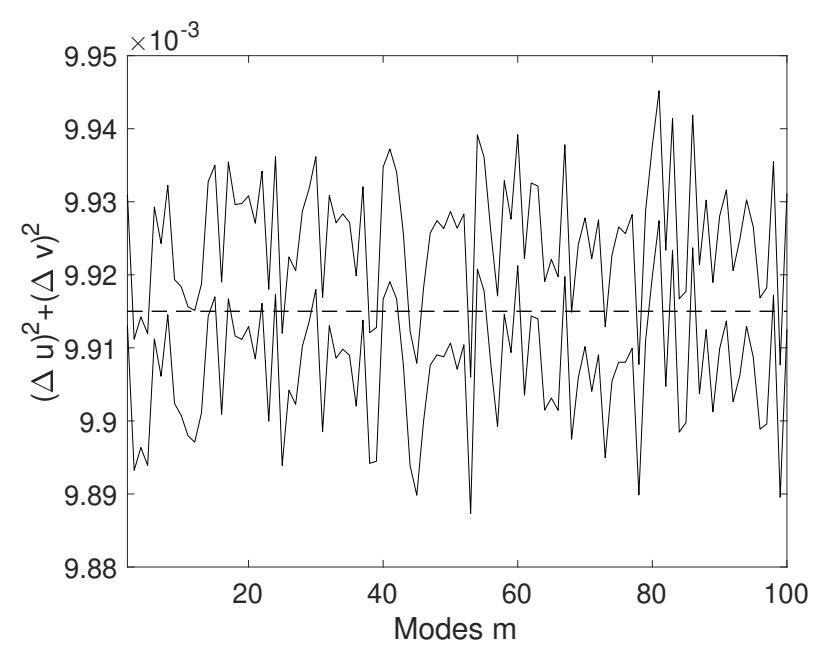

Figure 7. Graph of simulated multipartite entanglement product against number of entangled modes, using the Wigner representation. Sample numbers were $1.2 \times 10^{6}$ with an input squeezing of $r=3$, using a unitary matrix and pure state inputs. The upper and lower solid lines are sampling errors, the dashed line the exact result. Sampling errors here are about $\pm 1.0 \times 10^{-5}$.

also confirms $M$-partite entanglement for all $M$. The proof of the latter inequality is given in [54], for full tripartite inseparability. The proofs for genuine $N$-partite entanglement, and for the first inequality involving a product, follow along similar lines, using the methods developed in [56. The detailed proofs of these threshold points will be given elsewhere [71.

The above inequalities suffice to confirm the $M$-partite entanglement of the fields created by the ideal network, but other methods of detection are also possible [55, 74, 75]. This is particularly true if one assumes pure or Gaussian states, or is interested to measure full $\mathrm{N}$ partite inseparability only [17, 55, 57, 74, 76].

Figure (7), shows the result of a Wigner simulation of multipartite entanglement, plotted against the number of input modes, for $r=3$ and $S=1.2 \times 10^{6}$ samples. The total ratio of $\chi^{2} / k=0.965<1$, for 99 data points, showing that the simulation is consistent with the analytic result. The simulation error bars are $O\left(10^{-5}\right)$. The threshold for the signature in this case is 0.0404 at $M=100$, so the criterion is satisfied.

This level of precision are not obtained for all phasespace methods. In a positive-P simulation of multipartite entanglement, otherwise identical to figure (7) the total chi-squared ratio was $\chi^{2} / k=0.98<1$, for 99 independent points, indicating agreement with the analytic result, but the sampling error bars were $\pm 2 \times 10^{-3}$, which is 200 times larger. Similar large errors are found for the Q-function. In both cases, one must add or subtract corrections to transform the variance to symmetric ordering, which leads to larger sampling errors.

Phase-space simulations can readily include losses, decoherence and inhomogeneity. These all impact the

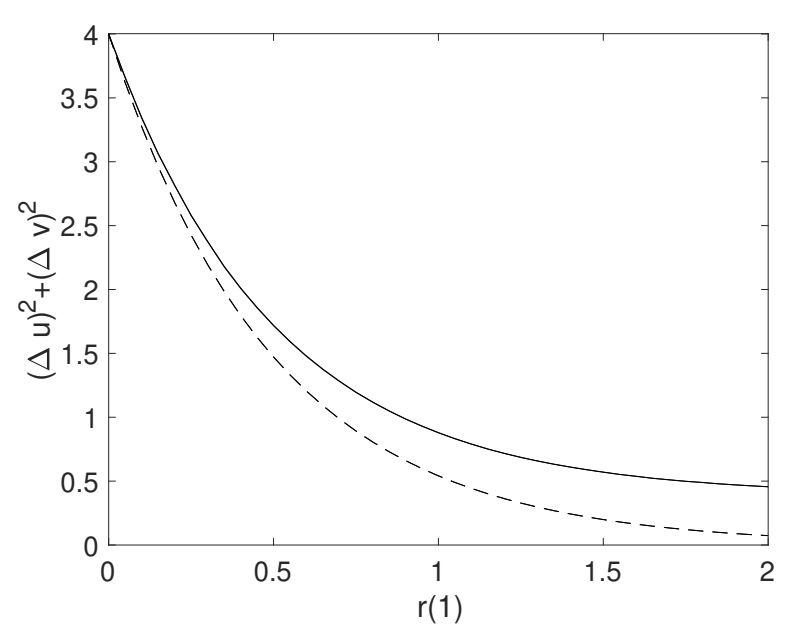

Figure 8. Graph of simulated multipartite entanglement product in the Wigner representation, versus squeezing $r$, with an input amplitude transmission of $t=0.95$ and $M=40$ modes. The required threshold of 0.44 is not reached even with a large squeezing of $r=2$. Other parameters as in Fig (7). Sampling errors are negligible: $\pm 10^{-6}$. This shows that input coupling losses can destroy the multi-partite entanglement signature.

amount of input squeezing required in realistic experiments. A simple example is shown in Fig (8) which simulates an input coupling amplitude transmission of 0.95 . This is sufficient to prevent the multipartite signature from being achieved for an $M=40$ network, with $r=2$.

\section{SUMMARY}

In summary, we have simulated Gaussian bosonic networks with phase-space methods. This efficiently simulates large networks with nonclassical inputs and decoherence. Up to $M=2^{14}=16,384$ modes were treated. There is excellent agreement with a recent 100-mode Gaussian boson sampling experiment for the total count probability, provided thermal decoherence is included. Other tests, including arbitrary order marginals, are also possible. The main limitation is that the phase-space sampling errors can be significant if the grouped probabilities are too small, but it is straightforward to increase sample numbers to reduce this. Similar limitations due to sampling error hold for the experimental data as well,

More generally, the representation used should be targeted to the measurement. Positive P-representations are optimal for normally ordered photo-detectors used in Gaussian boson sampling, while Wigner representations scale better for quadrature measurements and entanglement. Following the submission of our work, preprints have appeared covering related topics [77, 78], including calculations of low order marginals of grouped distributions, and improved direct sampling methods. 


\section{ACKNOWLEDGMENTS}

PDD thanks Jian-Wei Pan for access to experimental data. Corrected results were downloaded on Oct 4, 2021. Large-scale calculations were performed on the OzSTAR national supercomputing facility funded by Swinburne University of Technology and the Australian National Collaborative Research Infrastructure Strategy (NCRIS). This work was also funded through an Australian Research Council Discovery Project Grant DP190101480, and a grant from NTT Research.

\section{APPENDIX: STATISTICS AND NUMERICAL VALIDATION}

\section{Statistical tests}

Statistical tests are essential in comparing theory to experimental data. In this paper, we compare phasespace simulations both with exactly known distributions, and with 100 mode experimental observations. The test procedures are similar in both cases. We use chi-square methods originally discovered by Pearson [67, which are widely used in probability and RNG validation [36, 68]. Other tests of probability difference are also feasible, since our techniques generate complete number distributions, but chi-square tests are preferable for sampled data because they take account of experimental sampling errors.

Chi-square tests are used to compare a theoretical probability distribution to a set of experimental measurements [67, and can also be used to compare two independent samples. In these tests, experimental observations are grouped into disjoint classes, with frequencies $f_{i}$ (for $i=1,2, \ldots, k)$, from $\mathcal{N}_{e}$ observations.

Let an hypothesis $\mathcal{H}$ give a probability $P_{i}$ for an observation in the $i$-th class. Defining an experimental probability estimate as $P_{i}^{e}=f_{i} / \mathcal{N}_{e}, \chi^{2}=$ $\sum_{i=1}^{k}\left(P_{i}^{e}-P_{i}\right)^{2} /\left(\sigma_{e, i}^{2}\right)$. This has a $\chi^{2}$ distribution with $\left\langle\chi^{2}\right\rangle / k=1$, provided the counts all have a nearly Gaussian distribution.

Here, $\sigma_{e, i}^{2}=P_{i} / \mathcal{N}_{e} \approx f_{i} / \mathcal{N}_{e}^{2}$ is the expected variance in the experimental counts, which have Poissonian fluctuations. To deal with small counts, it is commonly recommended that these should not be included if $f_{i}<f_{i}^{\text {min }}$. Knuth [36] suggests $f_{i}^{\text {min }}=20$, and $f_{i}^{\text {min }}=5$ is recommended by NIST [68, 79]. We take the middle ground, ignoring counts less that $f_{i}^{\text {min }}=10$. Changing this threshold has little effect.

The true theoretical probability $P_{i}$ is not always available. In this work, we use an estimated value from phasespace simulations, which converges to $P_{i}$ in the limit of a large ensemble. The theoretical probability is estimated numerically from its ensemble mean, $\bar{P}_{i}$.

To obtain an error estimate for $\bar{P}_{i}$, it is computed numerically 33 by using $\mathcal{N}_{s} \gg 1$ sub-ensembles, each with many samples. From the central limit theorem, subensemble means are nearly Gaussian distributed, with a standard deviation of $\sigma_{s, i}$. These are obtained from the simulations. As a result, the simulated ensemble mean $\bar{P}_{i}$ has a standard deviation in the mean of $\bar{\sigma}_{s, i}=\sigma_{s, i} / \sqrt{\mathcal{N}_{s}}$.

This uncertainty in the true probability $P_{i}$ implies that the chi-squared test must be modified, which is similar the well-known case of two samples drawn from the same population 35, 80. For a finite ensemble, we employ an error measure of:

$$
\chi_{s}^{2}=\sum_{i=1}^{k} z_{i}^{2}=\sum_{i=1}^{k} \frac{\left(\bar{P}_{i}-P_{i}^{e}\right)^{2}}{\sigma_{i}^{2}} .
$$

This uses the fact that the experimental and simulated data are independent and nearly Gaussian. The difference in their means has a variance of $\sigma_{i}^{2}=\sigma_{e, i}^{2}+\bar{\sigma}_{s, i}^{2}$, which is obtained by adding the two variances. Correlated fluctuations modify the effective degrees of freedom, so we do not calculate the detailed $\chi_{s}^{2}$ distribution. However, since $\lim _{\mathcal{N}_{e}, \mathcal{N}_{s} \rightarrow \infty}\left\langle\chi_{s}^{2}\right\rangle / k=1$, we check if $\chi_{s}^{2} / k \sim O(1)$.

Fluctuations in the simulated data vanish in the limit of a large simulation, because $\bar{\sigma}_{s, i}^{2} \rightarrow 0$ as $\mathcal{N}_{s} \rightarrow \infty$. Such tests can be applied to any experimental probability, provided the measured data is binned to give enough counts to be significant. This requirement also includes marginal distributions which are included in our general definition.

Binned tests are also used in other RNG tests 68, which have very similar requirements. The difference between our tests and other RNG tests is that the comparisons are obtained through sampling. This is necessary because the exact Torontonian is non-computable. However, it does raise the question of how many samples are needed. This is answered by increasing the sample number until $\bar{\sigma}_{s, i}<\sigma_{e, i}$. We found that $1.2 \times 10^{6}$ was sufficient, using 1200 sub-ensembles of 1000 samples.

\section{Numerical validation tests}

To test our numerical results, independent numerical codes for simulations were written for two different languages (Matlab and Python) and computational platforms (a 14 core desktop, and a supercomputer with GPU hardware). Simulations were checked against known Torontonians for 16-mode networks with squeezed inputs [11, 81]. The 100 mode, million sample phase-space simulations took $\sim 100 \mathrm{~s}$ on a current desktop computer.

We validated the theoretical code in larger cases by comparison to exact analytic results for squeezed, thermalized and thermal inputs. Both unitary and lossy transmission matrices were used, and homogeneous or inhomogeneous squeezed inputs. For $40 \times 40$ and $100 \times 100$ matrices, 9 different types of moment were tested with up to four-dimensional binning. 


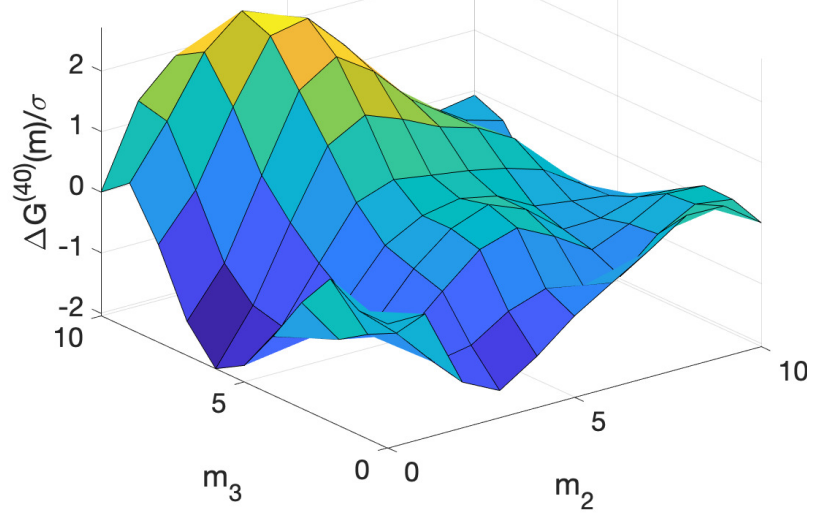

Figure 9. Normalized difference of simulation versus test distribution, for a four-fold partition and a thermal input. Results are for $\Delta \mathcal{G}_{(10,10,10,10)}^{(40)}(\boldsymbol{m}) / \sigma_{\boldsymbol{m}}$ vs $\boldsymbol{m}$, with sample numbers of $1.2 \times 10^{6}$. Data is given as a two-dimensional planar slice in $\left(m_{2}, m_{3}\right)$ of a four-dimensional probability space, with $m_{1}=6$ and $m_{4}=5$. No cut off was required in this slice.
A typical example output is plotted in Fig (9), which shows a test for a thermalized input with $r=\epsilon=1$ and $n=m=40$, using a random unitary transmission matrix. The output is the probability for a $40-t h$ order correlation, binned four ways, to give $11^{4}=14641$ click patterns. The graph is a two-dimensional slice in the $m_{2}-m_{3}$ plane, with $m_{1}=6$ and $m_{4}=5$, of the normalized error.

Plotted data was all within $\pm 2 \sigma$. The overall $\chi^{2}$ test gave $\chi^{2} / k=0.99$ in 9935 significant data points $(P>$ $10^{-7}$ ), out of 14641 possible click patterns. These results show complete agreement with the analytic probability model.

For each matrix, 68 distinct tests with up to $10^{4}$ data points were carried out, in $\mathrm{P}, \mathrm{Q}$ and Wigner phase-space. Probability cutoffs were used of $\mathcal{G}>10^{-7}$, with $1.2 \times 10^{6}$ total samples, since small probabilities are non-Gaussian. This effect is reduced by increasing the ensemble size. The overall result for $100 \times 100$ matrices was $\chi_{s}^{2} / k=$ $1.2 \pm 0.2$. This agrees with analytic tests, with evidence for nearly Gaussian errors.
[1] S. Scheel, in Quantum Information Processing, edited by T. Beth and G. Leuchs (Wiley-VCH, Weinheim, 2005) Chap. 28, pp. 382-392.

[2] S. Aaronson, Proceedings of the Royal Society of London A: Mathematical, Physical and Engineering Sciences 467, 3393 (2011).

[3] M. A. Broome, A. Fedrizzi, S. Rahimi-Keshari, J. Dove, S. Aaronson, T. C. Ralph, and A. G. White, Science 339, 794 (2013).

[4] A. Crespi, R. Osellame, R. Ramponi, D. J. Brod, E. F. Galvao, N. Spagnolo, C. Vitelli, E. Maiorino, P. Mataloni, and F. Sciarrino, Nature photonics 7, 545 (2013).

[5] M. Tillmann, B. Dakić, R. Heilmann, S. Nolte, A. Szameit, and P. Walther, Nature photonics 7, 540 (2013).

[6] J. B. Spring, B. J. Metcalf, P. C. Humphreys, W. S. Kolthammer, X.-M. Jin, M. Barbieri, A. Datta, N. Thomas-Peter, N. K. Langford, D. Kundys, et al., Science 339, 798 (2013).

[7] N. Spagnolo, C. Vitelli, M. Bentivegna, D. J. Brod, A. Crespi, F. Flamini, S. Giacomini, G. Milani, R. Ramponi, P. Mataloni, et al., Nature Photonics 8, 615 (2014).

[8] A. Crespi et al., Nat. Commun. 7, 10469 (2016).

[9] A. P. Lund, A. Laing, S. Rahimi-Keshari, T. Rudolph, J. L. O'Brien, and T. C. Ralph, Phys. Rev. Lett. 113, 100502 (2014).

[10] C. S. Hamilton, R. Kruse, L. Sansoni, S. Barkhofen, C. Silberhorn, and I. Jex, Phys. Rev. Lett. 119, 170501 (2017)

[11] N. Quesada, J. M. Arrazola, and N. Killoran, Physical Review A 98, 062322 (2018).

[12] R. Kruse, C. S. Hamilton, L. Sansoni, S. Barkhofen, C. Silberhorn, and I. Jex, Physical Review A 100, 032326 (2019).

[13] C. M. Caves, Physical Review D 23, 1693 (1981).
[14] L. McCuller, C. Whittle, D. Ganapathy, K. Komori, M. Tse, A. Fernandez-Galiana, L. Barsotti, P. Fritschel, M. MacInnis, F. Matichard, K. Mason, N. Mavalvala, R. Mittleman, H. Yu, M. E. Zucker, and M. Evans, Physical Review Letters 124, 171102 (2020).

[15] K. R. Motes, J. P. Olson, E. J. Rabeaux, J. P. Dowling, S. J. Olson, and P. P. Rohde, Phys. Rev. Lett. 114, 170802 (2015).

[16] Z.-E. Su, Y. Li, P. P. Rohde, H.-L. Huang, X.-L. Wang, L. Li, N.-L. Liu, J. P. Dowling, C.-Y. Lu, and J.-W. Pan, Phys. Rev. Lett. 119, 080502 (2017).

[17] M. Chen, N. C. Menicucci, and O. Pfister, Phys. Rev. Lett. 112, 120505 (2014).

[18] J. Roslund, R. M. De Araujo, S. Jiang, C. Fabre, and N. Treps, Nature Photonics 8, 109 (2014).

[19] J.-i. Yoshikawa, S. Yokoyama, T. Kaji, C. Sornphiphatphong, Y. Shiozawa, K. Makino, and A. Furusawa, APL Photonics 1, 060801 (2016).

[20] A. Marandi, Z. Wang, K. Takata, R. L. Byer, and Y. Yamamoto, Nature Photonics 8, 937 (2014).

[21] T. Inagaki, Y. Haribara, K. Igarashi, T. Sonobe, S. Tamate, T. Honjo, A. Marandi, P. L. McMahon, T. Umeki, K. Enbutsu, et al., Science 354, 603 (2016).

[22] Y. Yamamoto, K. Aihara, T. Leleu, K.-i. Kawarabayashi, S. Kako, M. Fejer, K. Inoue, and H. Takesue, npj Quantum Information 3, 1 (2017).

[23] H.-S. Zhong, H. Wang, Y.-H. Deng, M.-C. Chen, L.-C. Peng, Y.-H. Luo, J. Qin, D. Wu, X. Ding, Y. Hu, et al., Science 370, 1460 (2020).

[24] M. J. Bremner, A. Montanaro, and D. J. Shepherd, Phys. Rev. Lett. 117, 080501 (2016).

[25] S. Boixo, S. V. Isakov, V. N. Smelyanskiy, R. Babbush, N. Ding, Z. Jiang, M. J. Bremner, J. M. Martinis, and H. Neven, Nature Physics 14, 595 (2018). 
[26] F. Arute, K. Arya, R. Babbush, D. Bacon, J. C. Bardin, R. Barends, R. Biswas, S. Boixo, F. G. Brandao, D. A. Buell, et al., Nature 574, 505 (2019).

[27] D. Shepherd and M. J. Bremner, Proceedings of The Royal Society A: Mathematical, Physical and Engineering Sciences 465, 1413 (2009).

[28] D. Hangleiter, M. Kliesch, J. Eisert, and C. Gogolin, Phys. Rev. Lett. 122, 210502 (2019).

[29] D. Phillips, M. Walschaers, J. Renema, I. Walmsley, N. Treps, and J. Sperling, Physical Review A 99, 023836 (2019).

[30] D. J. Brod, E. F. Galvão, A. Crespi, R. Osellame, N. Spagnolo, and F. Sciarrino, Advanced Photonics 1, 034001 (2019).

[31] N. Quesada and J. M. Arrazola, Physical Review Research 2, 023005 (2020).

[32] Y. Li, M. Chen, Y. Chen, H. Lu, L. Gan, C. Lu, J. Pan, H. Fu, and G. Yang, arXiv preprint arXiv:2009.01177 (2020).

[33] B. Opanchuk, L. Rosales-Zárate, M. D. Reid, and P. D. Drummond, Physical Review A 97, 042304 (2018).

[34] H. Qi, D. J. Brod, N. Quesada, and R. García-Patrón, Physical review letters 124, 100502 (2020).

[35] K. Pearson, Biometrika 8, 250 (1911).

[36] D. E. Knuth, Art of computer programming, volume 2: Seminumerical algorithms (Addison-Wesley Professional, 2014).

[37] J. F. Corney, J. Heersink, R. Dong, V. Josse, P. D. Drummond, G. Leuchs, and U. L. Andersen, Physical Review A 78, 023831 (2008).

[38] H. P. Yuen, Physical Review A 13, 2226 (1976).

[39] P. D. Drummond and Z. Ficek, eds., Quantum Squeezing (Springer-Verlag, Berlin, Heidelberg, New York, 2004).

[40] H. Vahlbruch, M. Mehmet, K. Danzmann, and R. Schnabel, Physical Review Letters 117, 110801 (2016).

[41] M. G. Raymer, P. D. Drummond, and S. J. Carter, Optics letters 16, 1189 (1991).

[42] P. D. Drummond and B. Opanchuk, Physical Review Research 2, 033304 (2020).

[43] H. Fearn and M. Collett, Journal of Modern Optics 35, $553(1988)$

[44] M. Hillery, R. F. O'Connell, M. O. Scully, and E. P. Wigner, Phys. Rep. 106, 121 (1984).

[45] E. Wigner, Phys. Rev. 40, 749 (1932).

[46] P. D. Drummond and C. W. Gardiner, J. Phys. A 13, 2353 (1980).

[47] B. Opanchuk, L. Rosales-Zárate, M. D. Reid, and P. D. Drummond, Optics Letters 44, 343 (2019).

[48] P. D. Drummond, C. W. Gardiner, and D. F. Walls, Phys. Rev. A 24, 914 (1981).

[49] P. D. Drummond and S. Chaturvedi, Physica Scripta 91, 073007 (2016).

[50] R. J. Glauber, Phys. Rev. 130, 2529 (1963).

[51] J. Janszky and A. V. Vinogradov, Physical review letters 64, 2771 (1990).

[52] J. E. Moyal, Mathematical Proceedings of the Cambridge Philosophical Society 45, 99 (1949).

[53] K. Husimi, Proc. Phys. Math. Soc. Jpn. 22, 264 (1940).

[54] P. van Loock and A. Furusawa, Physical Review A 67, 052315 (2003).

[55] J. Sperling and W. Vogel, Physical review letters 111, 110503 (2013).
[56] R. Y. Teh and M. D. Reid, Physical Review A 90, 062337 (2014).

[57] A. Coelho, F. Barbosa, K. Cassemiro, A. Villar, M. Martinelli, and P. Nussenzveig, Science 326, 823 (2009).

[58] S. Armstrong, M. Wang, R. Y. Teh, Q. Gong, Q. He, J. Janousek, H.-A. Bachor, M. D. Reid, and P. K. Lam, Nature Physics 11, 167 (2015).

[59] W. H. Louisell, Quantum statistical properties of radiation (Wiley, New York, 1973).

[60] P. D. Drummond and M. Hillery, The quantum theory of nonlinear optics (Cambridge University Press, 2014).

[61] K. E. Cahill and R. J. Glauber, Physical Review 177, $1882(1969)$.

62] M. Olsen and A. Bradley, Optics Communications 282, 3924 (2009).

[63] C. Gardiner, Handbook of Stochastic Methods for Physics, Chemistry, and the Natural Sciences, Springer complexity (Springer, 2004).

[64] P. D. Drummond, B. Opanchuk, L. Rosales-Zárate, M. D. Reid, and P. J. Forrester, Physical Review A 94, 042339 (2016).

[65] J. Sperling, W. Vogel, and G. S. Agarwal, Phys. Rev. A 85, 023820 (2012).

[66] C. M. Caves and P. D. Drummond, Reviews of Modern Physics 66, 481 (1994).

[67] K. Pearson, The London, Edinburgh, and Dublin Philosophical Magazine and Journal of Science 50, 157 (1900).

[68] A. L. Rukhin, J. Soto, J. R. Nechvatal, M. E. Smid, E. B. Barker, S. D. Leigh, M. Levenson, M. Vangel, D. L. Banks, et al., A statistical test suite for random and pseudorandom number generators for cryptographic applications (2010).

[69] S. Aaronson and A. Arkhipov, Quantum Info. Comput. 14, 1383 (2014).

[70] M. D. Reid, Phys. Rev. A 40, 913 (1989).

[71] A. Dellios, P. D. Drummond, B. Opanchuk, R. Y. Teh, and M. D. Reid (2021).

[72] R. Y. Teh, M. Gessner, M. D. Reid, and M. Fadel, arXiv preprint arXiv:2108.06926 (2021).

[73] P. A. M. Dirac, Rev. Mod. Phys. 17, 195 (1945).

[74] S. Gerke, J. Sperling, W. Vogel, Y. Cai, J. Roslund, N. Treps, and C. Fabre, Physical review letters 114, 050501 (2015).

[75] L. K. Shalm, D. R. Hamel, Z. Yan, C. Simon, K. J. Resch, and T. Jennewein, Nature Physics 9, 19 (2013).

[76] A. S. Villar, L. S. Cruz, K. N. Cassemiro, M. Martinelli, and P. Nussenzveig, Physical Review Letters 95, 243603 (2005)

[77] J. F. Bulmer, B. A. Bell, R. S. Chadwick, A. E. Jones, D. Moise, A. Rigazzi, J. Thorbecke, U.-U. Haus, T. Van Vaerenbergh, R. B. Patel, et al., arXiv preprint arXiv:2108.01622 (2021).

[78] B. Villalonga, M. Y. Niu, L. Li, H. Neven, J. C. Platt, V. N. Smelyanskiy, and S. Boixo, arXiv preprint arXiv:2109.11525 (2021).

[79] J. T. Roscoe and J. A. Byars, Journal of the American Statistical Association 66, 755 (1971).

[80] K. Pearson, Biometrika , 457 (1932).

[81] B. Gupt, J. M. Arrazola, N. Quesada, and T. R. Bromley, Quantum Information Processing 19, 1 (2020). 